\title{
Excellent fosfomycin and linezolid susceptibility among multidrug resistant urinary Enterococcus isolates in Eastern India
}

\author{
Mallika Sengupta ${ }^{1}$, Riya Sarkar ${ }^{2}$, Soma Sarkar ${ }^{3}$, Sougata Ghosh ${ }^{4}$, Parthajit Banerjee ${ }^{5}$ and Manideepa \\ Sengupta $6, *$
}

\begin{abstract}
${ }^{1}$ Assistant Professor, Department of Microbiology, KPC Medical College, Kolkata, India. ${ }^{2}$ Senior Resident, Intermediate Reference Laboratory for Tuberculosis, Kolkata, India.

${ }^{3}$ Associate Professor, Department of Microbiology, NRS Medical College, Kolkata, India.

${ }^{4}$ Professor, Department of Microbiology, Medical College, Kolkata, India.

${ }_{5}^{5}$ Professor \& Head, Department of Microbiology, KPC Medical College, Kolkata, India.

${ }^{6}$ Professor \& Head, Department of Microbiology, Medical College, Kolkata, India.
\end{abstract}

Publication history: Received on 20 August 2020; revised on 27 August 2020; accepted on 30 August 2020

Article DOI: https://doi.org/10.30574/wjarr.2020.7.2.0318

\begin{abstract}
Urinary tract infection (UTI) is one of the most common infectious diseases. Enterococcus spp. is an important causative agent of UTI. Emergence of resistance is high among enterococcus isolates. Fosfomycin has emerged as a novel oral therapeutic option for urinary isolates. A prospective study was done in Medical College, Kolkata for a period of 6 months (from January to June 2018). After obtaining ethical clearance from the Institutional Review Board (IRB) urine samples with significant growth of enterococcus were included in the study. Identification of Enterococcus species was preliminary done by Gram stain and conventional biochemical tests along with automated identification by VITEK 2 Compact. These isolates were tested for antimicrobial susceptibility to different antibiotics like ampicillin/ penicillin, tetracycline, ciprofloxacin, levofloxacin, vancomycin, teicoplanin, linzolid, fosfomycin and nitrofurantoin by Kirby Bauer disc diffusion method and minimum inhibitory concentration (MIC) by VITEK 2 Compact. MIC for fosfomycin was done by E-test method. Interpretation of susceptibility was done according to the Clinical and Laboratory Standards Institute (CLSI) 2017 guidelines. During the period of 6 months (from January to June 2018) there were 194 isolates of Enterococcus spp. from 2732 urine samples showing a prevalence of 7.1\%. Among these 194 isolates there were 148 Enterococcus faecalis, 40 Enterococcus faecium, 2 Enterococcus casseliflavus, 2 Enterococcus gallinarum and 2 Enterococcus avium. All isolates were highly susceptible to vancomycin, teicoplanin, linezolid and fosfomycin. There were total of $8(4.12 \%)$ isolates of vancomycin resistant enterococcus. Resistance to ampicillin and high level gentamicin was significantly related in Enterococcus faecium. Fosfomycin is a highly susceptible for urinary enterococcus isolates. However, larger prospective in vivo studies are required to determine the efficacy.
\end{abstract}

Keywords: Antimicrobial susceptibility; Resistance; Urine; Fosfomycin; Linezolid

\section{Introduction}

Urinary tract infection (UTI) is one of the most common clinical entities encountered by the medical practitioners. The most frequent pathogens associated with UTI are Escherichia coli, Klebsiella pneumoniae, Enterococcus spp., Pseudomonas aeruginosa, Enterobacter spp., and Staphylococcus spp. (1). The genus enterococcus consists of Grampositive, facultatively anaerobic organisms that are ovoid in shape, arranged in short chains or in pairs. They were earlier classified as Group D Streptococcus (2). The emergence of resistance to the most common anti-enterococcal antibiotics which include the $\beta$-lactam antibiotics like ampicillin, high level aminoglycosides besides being inherently resistant to many others like cephalosporins and clindamycin has made the treatment of these infections a real

\footnotetext{
${ }^{*}$ Corresponding author: Manideepa Sengupta

Professor \& Head, Department of Microbiology, Medical College, Kolkata.
} 
challenge for clinicians (3). Enterococcus has intrinsic and acquired resistance to many antimicrobials. They have intrinsic resistance against a number of antimicrobials due to carrying several resistance genes as well as acquired resistance against several antibiotics like macrolides, vancomycin, tetracycline, and fluoroquinolones, resulting from either DNA mutation or acquisition of new genes through gene transfer (4). Multidrug resistant isolates are those isolate which are resistant to three or more different classes of antimicrobials (5).

Glycopeptide antibiotics are used in the treatment of infections caused by resistant enterococcus. Six types of glycopeptide resistance have been described in enterococci that can be distinguished on the basis of the sequence of the structural gene for the resistance ligase (vanA, vanB, vanC, vanD, vanE, and vanG) (6). In a study by Phukan et al, it was found that $24 \%$ of the enterococcus isolates were resistant to vancomycin (7). The emergence of high level resistance to aminoglycosides has made the therapeutic combination of penicillin and gentamicin ineffective. In a study done in Assam, high level gentamicin resistance (HLGR) and high level streptomycin resistance (HLSR) were found to be 53.76 and 33.33 per cent respectively (8).

Fosfomycin is a bactericidal antibiotic agent. It inhibits an enzyme-catalyzed reaction in the first step of the synthesis of the bacterial cell wall. Fosfomycin interferes with the first cytoplasmic step of bacterial cell wall biosynthesis, the formation of the peptidoglycan precursor UDP $N$-acetylmuramic acid (UDPMurNAc). This inhibitory action takes place at a step earlier than the action of beta-lactams or glycopeptides. Fosfomycin reduces adherence of bacteria to urinary epithelial cells. Fosfomycin is considerably active against both Gram negative and Gram positive pathogens. Specifically, fosfomycin is considered active against Enterococcus spp. (9).

The aim of this study was to determine the prevalence, identification, characterization and susceptibility pattern of enterococcus isolates from urine samples.

\section{Material and methods}

A prospective study was done in Medical College, Kolkata for a period of 6 months (from January to June 2018). The study was done after obtaining ethical clearance from the Institutional Review Board (IRB) of the college.

The uncentrifuged urine samples received in the laboratory were examined microscopically for presence of pus cells, bacteria, casts and other sediments. Semi quantitative urine culture was done for all samples as per standard criteria. After culture, colony count and identification of the significant urinary isolates were done by using standard microbiological techniques. The urine samples with significant growth of enterococcus were included in the study. Identification of enterococcus species was preliminary done by Gram stain, non-fastidious growth and conventional biochemical tests like catalase test, growth on $6.5 \% \mathrm{NaCl}$, MacConkey agar, bile esculin agar and arginine hydrolysis for genus identification and fermentation of mannitol, arabinose, sorbitol and growth on tellurite agar and automated identification by VITEK 2 Compact (BioMerieux Inc., France) for species identification.

Antimicrobial susceptibility test - These isolates were further tested for antimicrobial susceptibility to different antibiotics like ampicillin $(10 \mu \mathrm{g})$, tetracycline $(30 \mu \mathrm{g})$, ciprofloxacin $(5 \mu \mathrm{g})$, levofloxacin $(5 \mu \mathrm{g})$, vancomycin $(30 \mu \mathrm{g})$, teicoplanin $(30 \mu \mathrm{g})$, linzolid $(30 \mu \mathrm{g})$, fosfomycin $(200 \mu \mathrm{g})$ and nitrofurantoin $(300 \mu \mathrm{g})$ by Kirby Bauer disc diffusion method using standard microbiological techniques on Mueller Hinton agar plates. Minimum inhibitory concentration (MIC) was tested by VITEK 2 Compact (BioMerieux Inc., France) for penicillin, tetracycline, ciprofloxacin, levofloxacin, vancomycin, teicoplanin, linzolid, and nitrofurantoin. Disc diffusion for fosfomycin was carried out on Mueller Hinton agar supplemented with $25 \mu \mathrm{g} / \mathrm{ml}$ G6P with $200 \mu \mathrm{g}$ discs. Minimum inhibitory concentration (MIC) for fosfomycin was tested by E-strip (BioMerieux Inc., France) on the same medium with drug concentrations ranging from 0.064 $1024 \mu \mathrm{g} / \mathrm{ml}$. All interpretation of susceptibility pattern was done according to the Clinical and Laboratory Standards Institute (CLSI) version 2017 guidelines. Susceptibility to high level gentamicin $(120 \mu \mathrm{g})$ was done by Kirby Bauer disc diffusion method and interpretation was done by using EUCAST guidelines version 2016. The quality control for antimicrobial susceptibility testing was done with Staphylococcus aureus ATCC 25923 for disc diffusion and Enterococcus faecalis ATCC 29212 for dilution method.

All data were entered in the excel spreadsheet (Microsoft Office, Redmond, Washington, USA). The geometric mean (GM) and the standard deviation (SD) were calculated using excel spreadsheet. The statistical analysis of the data was done using STATA version 20. The data were summarized using mean along with standard deviation for continuous variables, and frequency along with percentages for categorical variables. Chi square test was used to check the categorical variables association and $p$ value $<0.05$ was taken as significant. 


\section{Results}

A total of 2732 urine samples were collected in the Department of Microbiology, Medical College, Kolkata during the period of 6 months (from January to June 2018). There were 194 isolates of Enterococcus spp. showing a prevalence of 7.1\%. Among these 194 isolates there were 148 (76.29\%) Enterococcus faecalis, 40 (20.62\%) Enterococcus faecium, 2 (1.03\%) Enterococcus casseliflavus, 2 (1.03\%) Enterococcus gallinarum and $2(1.03 \%)$ Enterococcus avium. Among the antimicrobial agents tested, 45 (23.19\%) isolates were susceptible ampicillin, 52 (26.8\%) isolates were susceptible and $6(3.09 \%)$ intermediately susceptible to ciprofloxacin, 54 (27.83\%) isolates were susceptible and 12 (6.18\%) intermediately susceptible to levofloxacin, 113 (58.25\%) were susceptible to high level gentamicin, 152 (78.35\%) were susceptible to nitrofurantoin and $37(19.07 \%)$ were susceptible to tetracycline (table 1). There were total of 8 (4.12\%) isolates of vancomycin resistant enterococcus which constitutes of 2 Enterococcus casseliflavus, 2 Enterococcus gallinarum 2 Enterococcus faecium and 2 Enterococcus faecalis. Only 4 isolates were resistant to teicoplanin. There was only 2 isolates resistant to fosfomycin. Only one isolate was intermediate susceptible to linezolid. Table 2 shows the relation between resistance and species of enterococcus. Resistance to ampicillin and high level gentamicin was significantly related in Enterococcus faecium. There were 140 (72.16\%) multidrug resistant (MDR) enterococcus which were resistant to three different classes of antimicrobials. All these MDR isolates were highly susceptible to linezolid and only 2 isolates were resistant to fosfomycin.

Table 1 Shows the antimicrobial susceptibility of Enterococcus spp. $(n=194)$

\begin{tabular}{|l|l|l|l|l|}
\hline Antimicrobial agent & $\begin{array}{l}\text { Enterococcus spp. } \\
(\mathbf{n = 1 9 4 )}\end{array}$ & $\begin{array}{l}\text { MIC range } \\
\boldsymbol{\mu g} / \mathbf{~ m l}\end{array}$ & $\begin{array}{l}\text { MIC90 } \\
\boldsymbol{\mu g} / \mathbf{~ m l}\end{array}$ & $\begin{array}{l}\mathbf{M I C}_{\mathbf{5 0}} \\
\boldsymbol{\mu g} / \mathbf{~ m l}\end{array}$ \\
\hline Ampicillin/ Penicillin & $45(23.19 \%)$ & $<=2->=32$ & 32 & 32 \\
\hline Ciprofloxacin & $52(26.8 \%)$ & $<=0.5->=8$ & 8 & 8 \\
\hline Levofloxacin & $54(27.83 \%)$ & $<=0.5->=8$ & 8 & 8 \\
\hline High level gentamicin & $113(58.25 \%)$ & - & - & - \\
\hline Nitrofurantoin & $152(78.35 \%)$ & $<=16-256$ & 128 & 16 \\
\hline Vancomycin & $186(95.87 \%)$ & $<=0.5->=32$ & 1 & 0.5 \\
\hline Teicoplanin & $190(97.94 \%)$ & $<=0.5->=32$ & 1 & 0.5 \\
\hline Linezolid & $193(99.48 \%)$ & $<=0.5-4$ & 1 & 0.5 \\
\hline Tetracycline & $37(19.07 \%)$ & $<=0.5->=16$ & 16 & 16 \\
\hline Fosfomycin & $192(98.97 \%)$ & $<=0.5-512$ & 8 & 2 \\
\hline
\end{tabular}

Table 2 Shows the relation of antimicrobial resistance to E. faecalis and E. faecium

\begin{tabular}{|l|l|l|l|}
\hline Antimicrobial agent & E. faecalis $(\mathbf{n = 1 4 8 )}$ & E. faecium (n=40) & P value \\
\hline Ampicillin & 110 & 39 & $\mathbf{0 . 0 0 0 7}$ \\
\hline Ciprofloxacin & 104 & 32 & 0.318 \\
\hline Levofloxacin & 98 & 30 & 0.342 \\
\hline High level gentamicin & 57 & 24 & $\mathbf{0 . 0 1 9}$ \\
\hline Nitrofurantoin & 15 & 9 & 0.058 \\
\hline Vancomycin & 2 & 2 & 0.199 \\
\hline Teicoplanin & 2 & 2 & 0.199 \\
\hline Tetracycline & 125 & 32 & 0.48 \\
\hline Fosfomycin & 1 & 1 & 0.381 \\
\hline
\end{tabular}




\section{Discussion}

In a study done by Chakroborty et al in Kolkata in 2011 there was a prevalence of 7.3\% enterococcus isolates from all clinical samples (10). In this study it was found that there is a prevalence of $7.1 \%$ among urine samples which is very similar to the previous finding.

In a study done in Assam, speciation of 93 enterococcus species by Vitek 2 automated system was similar to that by conventional biochemical tests. E. faecalis was the commonest species $(81.72 \%)$ isolated, followed by E. faecium $(12.9 \%)$, E. raffinosus $(3.23 \%, \mathrm{n}=3)$, E. avium $(1.08 \%, \mathrm{n}=1)$ and E. gallinarum $(1.08 \%, \mathrm{n}=1)(8)$. In this study, among these 194 isolates there were 148 (76.29\%) Enterococcus faecalis, 40 (20.62\%) Enterococcus faecium, 2 (1.03\%) Enterococcus casseliflavus, 2 (1.03\%) Enterococcus gallinarum and 2 (1.03\%) Enterococcus avium. Similar results were seen in another study in Uttar Pradesh where it was found that out of 100 Enterococcus strains 47 were E. faecalis, 51 were E. faecium, 2 were E. gallinarum and 1 was E. casseliflavus (11).

Glycopeptide resistance in enterococci is associated with diverse phenotypes. Intrinsic low-level vancomycin resistance is characteristic of Enterococcus gallinarum and Enterococcus casseliflavus/flavescens. The VanC-1 ligase is specific for E. gallinarum, and the VanC-2/3 ligase is specific for E. casseliflavus/flavescens. The VanC enzymes participate in the synthesis of pentapeptide peptidoglycan precursors ending in D-alanyl-D-serine, which display reduced affinity for vancomycin. Organisms with resistance to VanC remain susceptible to teicoplanin. This naturally occurring vancomycin resistance has not been shown to be transferable, and the related genes are chromosomally encoded in the members of these species (12). In this study there were two isolates each of E. gallinarum and E. casseliflavus which were resistant to vancomycin but susceptible to teicoplanin.

In this study the prevalence of multidrug resistant enterococcus was $72.16 \%$. This is similar to the finding of Bhatt et al where the prevalence of multidrug resistance among enterococcus was found to be $63 \%$ among 200 clinical isolates (13). In a study done by Praharaj and colleagues out of 367 isolates of enterococcus 32 (8.7\%) were found to be resistant to vancomycin. None of the Enterococcus isolates were resistant to linezolid (14). However in this study there were 8 $(4.12 \%)$ isolates of vancomycin resistant enterococcus. This finding is less in comparison to the findings in Mangalore where out of 150 total isolates, 13 (8.6\%) isolates showed vancomycin resistance, of which 11(7.3\%) had an MIC >8 $\mu \mathrm{g} / \mathrm{ml}(15)$. Though the first report of linezolid resistant enterococcus isolate was from Kolkata (16), we did not find any linezolid resistant strain. Only one isolate of Enterococcus faecium was intermediate susceptible to linezolid with MIC of $4 \mu \mathrm{g} / \mathrm{ml}$. In a study done in Katihar, 2.8\% enterococcus isolates were resistant to linezolid (17).

In a study by Adhikari et al, the highest resistance to aminoglycoside was observed among E. faecium, followed by $E$. durans, E. fecalis and E. casseliflavus, both by disk-diffusion and agar-screen methods. The high-level aminoglycoside resistance (HLAR) was significantly $(P<0.05)$ higher in E. faecium (18). In this study it was found that resistance to ampicillin and high level gentamicin was significantly related in Enterococcus faecium. In this study 81 (41.75\%) enterococcus were resistant to high level gentamicin. In a study done in Iran among 53 isolates, high-level gentamicin resistance was observed among $50.9 \%$ of the isolates though all of the isolates were MDR (100\%) (19). In another study, it was found that out of 100 isolates studied $93 \%$ of isolates were resistant to one or more antimicrobial agents, with the most frequent resistance found against tetracycline (86\%), ciprofloxacin (73\%) and quinupristin-dalfopristin (53\%). High level gentamicin and high level streptomycin resistance were detected in 50\% and 34\% of isolates, respectively (20). There were only two isolates resistant to fosfomycin. Urinary enterococcus showed excellent susceptibility to fosfomycin (98.97\%) and linezolid (99.48\%) and good susceptibility to nitrofurantoin (78.35\%). Hence these drugs are commonly used for management of UTI. Molecular characterization of the vancomycin resistant enterococcus and high level aminoglycoside resistance among the isolates was beyond the scope of this study.

\section{Conclusion}

Urinary enterococcus isolates are highly susceptible to fosfomycin and linezolid. Resistance to ampicillin and high level gentamicin was significantly related in Enterococcus faecium. However, larger prospective in vivo studies are required to determine the efficacy.

\section{Compliance with ethical standards}

\section{Disclosure of conflict of interest}

The authors declare no conflict of interest. 


\section{References}

[1] Bennett JE, Dolin R, Blaser MJ, Mandell, Douglas. Bennett's principles and practice of infectious diseases. Eighth edition. Philadelphia, PA: Elsevier/Saunders. 2015; 2.

[2] Murray BE. The life and times of the Enterococcus. ClinMicrobiol Rev. 1990; 3(1):46-65.

[3] Gupta V, Singla N, Behl P, Sahoo T, Chander J. Antimicrobial susceptibility pattern of vancomycin resistant enterococci to newer antimicrobial agents. Indian J Med Res. 2015 Apr; 141(4):483-6.

[4] Esmail MAM, Abdulghany HM, Khairy RM. Prevalence of Multidrug-Resistant Enterococcus faecalis in HospitalAcquired Surgical Wound Infections and Bacteremia: Concomitant Analysis of Antimicrobial Resistance Genes. Infect Dis [Internet]. 2019 Oct 15 Jun 26; 12.

[5] Falagas ME, Karageorgopoulos DE. Pandrug Resistance (PDR), Extensive Drug Resistance (XDR), and Multidrug Resistance (MDR) among Gram-Negative Bacilli: Need for International Harmonization in Terminology. Clin Infect Dis. 2008 Apr 1; 46(7):1121-2.

[6] Depardieu F, Perichon B, Courvalin P. Detection of the van alphabet and identification of enterococci and staphylococci at the species level by multiplex PCR. J ClinMicrobiol. 2004 Dec; 42(12):5857-60.

[7] Phukan C, Lahkar M, Ranotkar S, Saikia KK. Emergence of vanA gene among vancomycin-resistant enterococci in a tertiary care hospital of North - East India. Indian J Med Res. 2016 Mar; 143(3):357-61.

[8] Barman J, Nath R, Saikia L. Drug resistance in Enterococcus species in a tertiary level hospital in Assam, India. Indian J Med Res. 2016 Jan; 143(1):107-10.

[9] Falagas ME, Vouloumanou EK, Samonis G, Vardakas KZ. Fosfomycin. ClinMicrobiol Rev. 2016 Apr; 29 (2):321-47.

[10] Chakraborty A, Pal N, Sarkar S, Gupta M. Antibiotic resistance pattern of Enterococci isolates from nosocomial infections in a tertiary care hospital in Eastern India. J Nat SciBiol Med. 2015; 6(2):394.

[11] Jaiswal S, Singh A, Verma RK, Singh DP, Kumari S. Characterization, speciation and antimicrobial resistance pattern of Enterococcus species isolated from clinical specimens at a rural tertiary care hospital. Int J Res Med Sci. 2017 Jul 26; 5(8):3484.

[12] Reid KC, Cockerill FR, Patel R. Clinical and Epidemiological Features of Enterococcus casseliflavus/flavescens and Enterococcus gallinarum Bacteremia: A Report of 20 Cases. Clin Infect Dis. 2001 Jun 1; 32(11):1540-6.

[13] Bhatt P, Patel A, Sahni AK, Praharaj AK, Grover N, Chaudhari CN, et al. Emergence of multidrug resistant enterococci at a tertiary care centre. Med J Armed Forces India. 2015 Apr; 71(2):139-44.

[14] Praharaj I, Sujatha S, Parija SC. Phenotypic \& genotypic characterization of vancomycin resistant Enterococcus isolates from clinical specimens. Indian J Med Res. 2013 Oct; 138(4):549-56.

[15] Fernandes SC, Dhanashree B. Drug resistance \& virulence determinants in clinical isolates of Enterococcus species. Indian J Med Res. 2013 May; 137(5):981-5.

[16] Kumar S, Bandyoapdhyay M, Chatterjee M, Mukhopadhyay P, Poddar S, Banerjee P. The first linezolid-resistant Enterococcus faecium in India: High level resistance in a patient with no previous antibiotic exposure. Avicenna J Med. 2014; 4(1):13.

[17] Biswas PP, Dey S, Adhikari L, Sen A. Detection of vancomycin resistance in enterococcus species isolated from clinical samples and feces of colonized patients by phenotypic and genotypic methods. Indian J PatholMicrobiol. 2016 Jun; 59(2):188-93.

[18] Adhikari L. High-level Aminoglycoside Resistance and Reduced Susceptibility to Vancomycin in Nosocomial Enterococci. J Glob Infect Dis. 2010; 2(3):231-5.

[19] Taji A, Heidari H, Ebrahim-Saraie HS, Sarvari J, Motamedifar M. High prevalence of vancomycin and high-level gentamicin resistance in Enterococcus faecalis isolates. ActaMicrobiolImmunol Hung. 2019 Jun 1; 66(2):203-17.

[20] Haghi F, Lohrasbi V, Zeighami H. High incidence of virulence determinants, aminoglycoside and vancomycin resistance in enterococci isolated from hospitalized patients in Northwest Iran. BMC Infect Dis. 2019 Aug 27; 19(1):744. 